\title{
MicroRNAs as Regulators of Cisplatin Resistance in Lung Cancer
}

\author{
Ying Chen Yanping Gao Kai Zhang Chen Li Yan Pan Jing Chen Rui Wang \\ Longbang Chen
}

Department of Medical Oncology, Jinling Hospital, School of Medicine, Nanjing University, Nanjing, China

\section{Key Words}

MicroRNA • Cisplatin $•$ Lung cancer $•$ Chemo resistance $\cdot$ Post-transcriptional regulation

\begin{abstract}
Cisplatin (CDDP) is one of the most effective broad-spectrum anticancer drugs, which has been employed for the treatment of lung cancer. The development of CDDP resistance is a major problem of tumor chemotherapy. MicroRNAs (miRNAs) are an abundant class of small nonprotein-coding RNAs, involved in the initiation and progression of human cancer. Increasing evidence has shown that dysregulation of miRNAs is involved in chemo resistance of tumor cells to anti-cancer drugs, including CDDP. This article summarizes current research involving miRNAs as regulators of key target genes for CDDP resistance in lung cancer. Potential use of targeting miRNAs can lead to miRNA-based therapies, which will be helpful for overcoming drug resistance and developing more effective personalized anti-cancer treatment strategies in human lung cancers.

\footnotetext{
Copyright (C) 2015 S. Karger AG, Basel
}

\section{Introduction}

Lung cancer account for about $13 \%$ of total cancer diagnoses and is the first cause of cancer-related death [1]. In terms of clinically and tumor genetics, lung cancer can be divided into small cell carcinoma (SCLC) and non-small cell carcinoma (NSCLC) [2]. Despite huge progresses have been achieved in lung cancer treatment, platinum agents remain an important component of chemotherapy for all stages of lung cancer. CDDP-based adjuvant chemotherapy has been established, because it can improve 5-years the survival rate in completely resected NSCLC tumors [3]. Meanwhile, CDDP-based treatment is also the golden

Rui Wang, MD

and Longbang Chen, MD
Department of Medical Oncology, Jinling Hospital, School of Medicine, No. 305 Zhongshan East Road, Nanjing 210002, Jiangsu Province, China University, Nanjing, Jiangsu 210002, (China)

Tel.+86-25-80860072, E-Mail wangrui1977218@163.com and chenlongbang@yeah.net 
standard for SCLC therapy, which cannot be overstepped by any chemotherapeutic strategies or newer cytotoxic agents [4]. CDDP is a well-known DNA-damaging cytotoxic agent, and it is currently thought that DNA platination is an essential first step in its cytotoxic activity. Once CDDP enters cells, platinum-DNA adducts are formed and the signals activate apoptotic pathway [5]. In recent years, much progress made in the treatment of lung cancer, CDDPbased chemotherapy also plays an important role. Unfortunately, the development of chemo resistance has inhibited curative effect in lung cancer [6].

CDDP resistance is such a significant clinical challenge in lung cancer patients. Therefore, it is necessary to elucidate the molecular mechanisms involved in CDDP resistance so as to overcome such resistance and develop novel, more efficacious treatments for lung cancer patients. Ultimately, molecular factors inducing resistance do so by inhibiting propagation of the DNA damage signal to the apoptotic machinery. Apoptotic inhibitor molecules, such as secretory clusterin (sCLU) and Akt, increase cellar resistance when overexpression [7].

The cytotoxic effects of CDDP on cells include multiple progresses, which contribute to complex chemo resistance mechanisms, such as drug transport, drug metabolism, DNA repair, cell survival, and apoptosis [5]. Acquired resistance to CDDP, an issue in cancer chemotherapy, has been demonstrated as a multifactorial nature [8]. CDDP-resistance can be induced by alterations to a huge number of intracellular pathways, where miRNAs play a vital role. MiRNAs, a family of non-coding RNAs with a length of 18-23 nucleotides, function by regulating gene expression via sequence-specific targeting of mRNAs [9]. The mature miRNAs and RNA-induced silencing complex (RISC) combined into RISC-miRNA complex to target mRNA, causes the degradation of mRNA and reduction of related function proteins expression. Also, miRNAs might bind to protein factors required for translation or alter mRNA secondary structure, inhibiting protein translation [10]. Experimental and clinical studies have revealed that miRNAs can function as tumor suppressor genes or oncogenes during the process of lung cancer development. For example, low expression of miR-409-3p is correlated with poorer tumor differentiation [11]. MiRNAs such as miR-138 and miR-4782$3 p$ may inhibit proliferation of NSCLC cells in vitro, and their overexpression is associated with favorable prognosis in NSCLC patients $[12,13]$. On the contrary, low expression of miR-4500 was demonstrated to favor tumor growth via targeting LIN28B and NRAS in NSCLC [14]. And, methylation-silencing of miR-9-3 and miR-193a may be a vital epigenetic mechanisms promoting lung cancer cell growth [15]. Mir-30c, another prognostic indicator associated with poor survival of patients, is reported to control lung cancer invasion through regulating MTA1 in NSCLC [16]. To date, there are numerous research findings alluding to miRNAs and their influence on the sensitivity of CDDP-based chemotherapy of lung cancer cells. It is demonstrated that a single miRNA can affect the expression of a wide variety of proteins [17]. Therefore, the ability of miRNAs to modulate the sensitivity of cancer cells to chemotherapy via post-transcriptionally regulating multiple target genes highlights the potential of manipulating relevant miRNAs to overcome chemo resistance in clinic.

Targeting dysregulated miRNAs may be a promising strategy for improving therapeutic efficiency or forecasting the responses of lung cancer patients to CDDP-based chemotherapy $[9,17]$ (Fig. 1). In this review, we provide an overview on the roles of miRNAs in the development of CDDP resistance in lung cancer.

\section{CDDP regulates miRNA expression}

\section{CDDP regulates miRNA transcription}

Modulation of the transcription of miRNA primary transcripts (pri-miRNAs) represents a major site at which the expression of mature miRNAs can be regulated [9]. Thus, transcription of pri-miRNAs can be subject to regulation by elements of the DDR (DNA damage response) pathway in the same manner as protein-coding genes following CDDP treatment. 


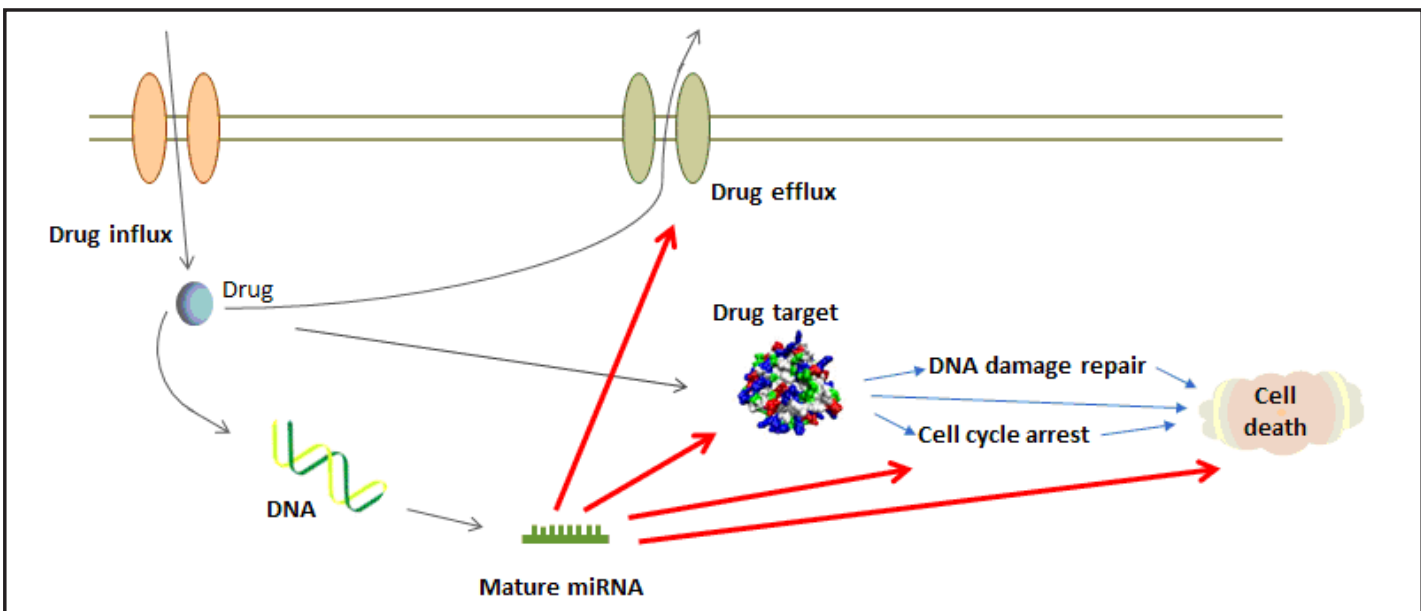

Fig. 1. Roles of miRNAs in anticancer drug resistance. Multiple cellular pathways involved in CDDP resistance of cancer cell include alteration of drug target, altered regulation of the cell cycle and apoptosis, increased DNA damage repair and ejection of the drug from the cell by drug efflux pumps. Dysregulation of certain miRNAs plays a critical role in these processes.

Fig. 2. Influence of CDDP treatment on the miRNA transcription. In early stage of DNA damage response in CDDP-treated tumor cells, activation of ATM suppresses phosphorylation of p53 by regulating the transcription of miRNA-34a and miRNA-128-2, which attenuates the anticancer activity of CDDP in turn.

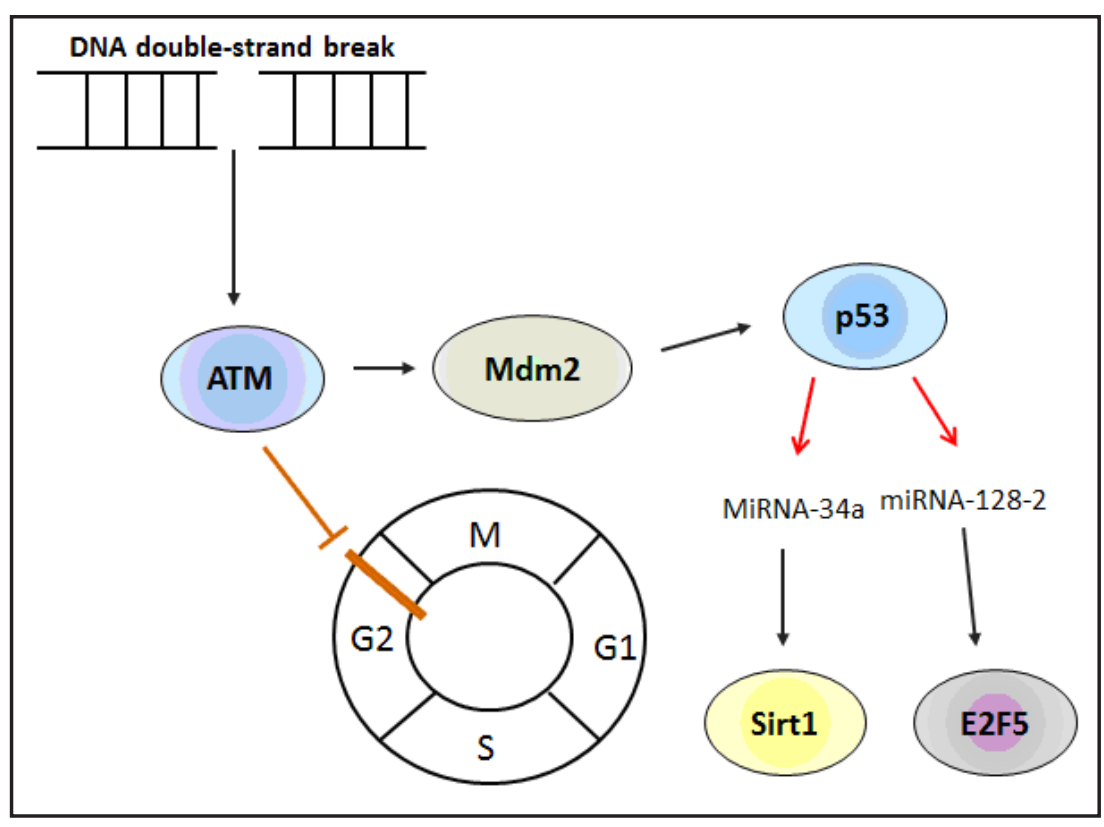

The mediation of p53 in the cellular toxic effects of CDDP is a direct consequence of DNA damage. CDDP preferentially activates the two kinases ATM (ataxia telangiectasia mutated protein) and ATR (ATM- and Rad3-related protein), which phosphorylates p53 to initiate activation of the p53 protein and then leads to checkpoint activation. Interestingly, CDDP also activates CHK2, which is a downstream target of ATM, but the effect of CDDP on CHK2 appears to be independent of ATM [3]. Also, ATM is reported to be involved in phosphorylation of p53 in early stage of DNA damage response [18]. ATM is activated within minutes after DNA double-strand break, and then phosphorylates MDM2 on multiple sites near its RING domain, causing selective inhibition of p53 poly-ubiquitination [19]. Recently, miR-34a has been found to be a direct transcriptional target of p53 [20]. For example, Wang et al. indicated that miR-34a was transcriptionally regulated by the tumor suppressor p53 in lung adenocarcinoma cell line (A549). In lung cancer cells treated with CDDP, miR-34a inhibits the cell growth through reducing the expression of its downstream target silent information regulator 1 (Sirt1) [21]. Meanwhile, the inhibition of SIRT1 by miR-34 family 
could lead to an increase in acetylated p53 and expression of p21 and PUMA, transcriptional targets of p53 which regulate the cell cycle and apoptosis [22]. Likewise, another member of miR-34 family, miR-34c, is demonstrated as a direct effector of p53 and its ectopic expression causes cell-cycle arrest [23].

In another study, Donzelli et al. showed that p53R175H, a hotspot p53 mutant, induces miRNA-128-2 expression in vitro. Mutant p53 binds to the putative promoter of miR128-2 host gene, ARPP21, determining a concomitant induction of ARPP21 mRNA and miR-1282. MiR-128-2 expression in lung cancer cells inhibits apoptosis and confers the increased resistance to CDDP treatments. At the molecular level, miR-128-2 post-transcriptionally targets E2F5 and leads to the abrogation of its repressive activity on p21waf1 transcription. p21waf1 protein localizes to the cytoplasmic compartment, where it exerts an anti-apoptotic effect by preventing pro-caspase-3 cleavage [24] (Fig. 2).

\section{CDDP regulates miRNA processing}

The biogenesis of miRNA is a multistep process, and each of these steps is potentially subjected to regulation, which can be regulated by DNA damage. Transcription factor E2F1 is a member of the E2F family of transcription factors, which plays a crucial role in the control of cell cycle and action of tumor suppressor proteins. E2F1, as a DNA damage responsive transcription factor, can also regulate some downstream target genes responsible for checkpoint activation, DNA repair, and apoptosis, participating in maintaining genomic integrity under DNA damage stress $[24,25]$. In CDDP-treated cells, E2F1 over-expression could up-regulate DROSHA mRNA and protein expression, which was accompanied by an increase of mature miR-630 rather than pri-miR-630, suggesting that E2F1 can enhance miR-630 biosynthesis under CDDP exposure through promoting DROSHA-mediated primiR-630 processing [25]. MiR-630 inhibits cell proliferation by targeting cell-cycle kinase 7 (CDC7), but maintains the apoptotic balance by targeting multiple activators of apoptosis under genotoxic stress. On the one hand, miR-630 promotes apoptosis via blocking CDC7mediated initiation of DNA synthesis; on the other hand, it reduces apoptosis by targeting several other apoptotic modulators such as PARP3, DDIT4, EP300 and EP300 downstream effector p53, thereby maintaining the apoptotic balance [26].

\section{Cispltain-related targets of miRNA}

\section{MiRNAs involved in cell cycle control of CDDP}

Cell cycle inhibition, currently being tested in clinical trials, might be antagonistic to CDDP and other cytotoxic drugs. Cyclin-dependent kinase, comprised of a catalytic Cdk subunit and a regulatory cyclin subunit, control cell cycle transition. Cyclin-Cdk is unique in each phase of cell cycle. Cyclin E can regulate the transit of cells from quiescence into $\mathrm{S}$ phase via activating Cdk2. Moreover, cyclin $\mathrm{E}$ has been found as an important regulator of the rate limiting for S phase entry and required in G1 progression. As the downstream effector of many cancer-associated pathways, Cyclin E-Cdk2 is often deregulated in cancer cells, which likely contributes to the development of cancer. Thus, cyclin E may serve as a potential molecular target in cancer therapy [27]. MiR-25, as an oncogene, overexpresses in lung cancer including SCLC and NSCLC. In recent research, Zhao et al. found that miR-25 was overexpressed in both SCLC cells and tumor tissues, and further testified that miR-25 down regulation could lead to CDDP chemo resistance in SCLC cells via inducing G0/G1 cell cycle arrest by targeting cyclin E2 [28].

Additionally, miRNAs which suppress G1/S cell cycle have been reported. CDK6, activated by D-type cyclin, is another miRNA target, and its expression can be reduced by miR-145 up regulation, which finally causes the inhibition of G1 progression, and antagonizes CDDP cytotoxicity [29]. Also, CDDP inhibits A549 cell growth by miR-98 regulating TP53 pathway [30]. TP53, as a checkpoint control protein that determines cellular fate upon DNA damages, can delay the progression of the cell cycle from G1 to S phase, thus allowing for repair of

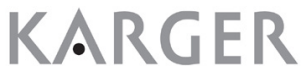


DNA damage $[31,32]$. The p21 $1^{\text {WAF1/CIP1 }}$ gene, which encodes a cyclin-dependent kinase inhibitor, may be critical for tumor suppressor gene p53-induced cell cycle arrest. Wang and his colleagues reported that miR-224 promotes the chemo resistance of human lung adenocarcinoma cells to cisplatin via regulating G1/S transition and apoptosis by targeting p2 $1^{\text {WAF1/CIP1 }}[33]$.

\section{MiRNAs involved in regulation of EMT}

Epithelial-mesenchymal transition (EMT) is an essential developmental process by which cells get increased migratory behavior by the loss of epithelial characteristics and the acquisition of a mesenchymal phenotype [34]. Emerging evidence suggests that EMT plays a crucial role in tumor metastasis, where tumor cells undergo a change to a more dedifferentiated state to acquire their invasive phenotype [35].

The miR-200 family has recently been shown to prevent EMT by suppressing the expression of ZEB1 and ZEB2, two transcriptional repressors of E-cadherin. E-cadherin expression has been proposed as a novel biomarker predicting the clinical activity of the EGFR inhibitor erlotinib in patients with NSCLC. It was also found that overexpression of miR-200c increases the sensitivity of bladder cancer to EGFR-inhibiting agents and restores the sensitivity of several types of cancer to antimicrotubule drugs. In CDDP-treated cells, the sensitizing effect of miR-200c transfection can be attributed to blockage of cell proliferation and induction of cancer cell death. The molecular mechanisms by which miR-200c regulates CDDP resistance of tumor cells are not well elucidated, and novel determinants of CDDP sensitivity (such as DNA repair enzymes or proteins with signaling transduction activity) specifically targeted by miR-200c could be potentially identified in future studies [35]. Overexpression of miRNA-17 family (miR-17, 20a and 20b) can not only decrease the sensitivity of CDDP but also reduce migration by reversing EMT phenotype in A549/DDP cells. These functions of miR-17, 20a, 20b may be caused at least in part via inhibition of TGF beta signal pathway, because those miRNAs are shown to directly target and repress TGFbeta receptor 2 (TGF beta R2) which is an important component of TGF beta signal pathway [36]. In another report, both miR-15b and miR-34a can regulate EMT and the response of lung adenocarcinoma cells to CDDP both in vitro and in vivo by targeting PEBP4 $[37,38]$. Also, miR-27a can regulate EMT via direct and functional targeting Raf-1 kinase inhibitory protein (RKIP), which then affects the sensitivity of lung adenocarcinoma cells to CDDP both in vitro and in vivo [39].

\section{MiRNAs involved in regulation of drug transports}

Previous studies have indicated that the decreased uptake of water-soluble drugs and enhanced drug efflux from cancer cells are the biochemical and cytological mechanisms cytological mechanisms of drug resistance in cancer cells. ATP-Binding Cassette (ABC), as a family of transmembrane, is important mediator of multidrug resistance (MDR) in patients with cancer. It is well known that $\mathrm{ABC}$ transporters can mediate the active efflux transport of a variety of structurally and mechanistically unrelated compounds across biological membranes of the cell and numerous organelles [40]. The ATP-binding cassette, sub-family B (MDR/TAP), member 9 (ABCB9), which is a transporter associated with antigen processinglike (TAPL), translocates a broad spectrum of peptides from the cytosol into the lumen of lysosomes [41]. A recent study has shown that miR-31 exerts an anti-apoptotic effect most likely through inhibition of $A B C B 9$ and its expression inversely correlates with the expression of the drug resistance gene ABCB9. In CDDP-resistant NSCLC cells, overexpression of miR-31 could lead to the decreased level of $A B C B 9$ protein, which induced the decreased uptake of CDDP and consequently the inhibition of CDDP-mediated apoptosis [42]. Another common form of chemo resistance is caused by activation of the MDR1 (ABCB1) gene, which can 
result in the overexpression of P-glycoprotein (P-gp). The up regulation of both miR-27a and miR-451 confer broad MDR to lung cancer cells through regulation of the expression of MDR1/P-glycoprotein [42]. Up-regulation of Let-7c sensitizes A549/DDP cells to CDDP by targeting ABCC2 [43]. Moreover, miR-10a, miR-106a and miR-134 can regulate different members of MDR to affect the responses of lung adenocarcinoma cells to CDDP [44-46].

Recent studies have shown that copper transporters are also involved in the transport of platinum. As a P-type ATPase copper transporter, ATP7A is expressed in many tissues, which plays crucial role in the modulation of platinum-resistance. An ATP-dependent DDP transporting activity of ATP7A is responsible for the decreased DDP accumulation. In resistant cells, ATP7A may be a potential target of miR-495. Song et al. has demonstrated that miR-495 regulates the CDDP resistance by modulation of ATP7A expression in NSCLC cells. MiR-495 increases the intracellular CDDP accumulation and causes overexpression of ATP7A. Then, drug concentration reducing decreases the CDDP efficiency [47]. KIT, also named as CD117, is a transmembrane protein receptor encoded by the c-kit proto-oncogene, which can be targeted by miR-137 in H446/CDDP cells [48, 49].

\section{MiRNA involved in regulation of CDDP-induced survival and /or apoptosis pathways}

The molecular mechanisms which underlie inhibition of apoptosis responsible for the acquired CDDP resistance includes loss of p53 function, reduced Fas expression, down regulation of Bax or Bad, up regulation of Bcl- 2 or Bcl-xL, suppressed caspase- 3 activity, HER-2/neu overexpression, enhanced activity of PI3K/Art, deregulated MAPK pathway, and so on [5]. A number of miRNAs have been reported to be involved in regulation of apoptosisrelated molecular signal pathways (Fig. 3). For example, the effect of miR-24-3p on ATG4A occurred directly through its 3'-UTR region, and is the inhibition of cell proliferation and promotion of apoptosis [50]. Also, it was reported that enforced miR-135a/b expression reduced MCL1 protein level and sensitized A549/CDDP cells to CDDP [51].

Members of the Bcl-2 family are key players in regulating apoptosis, comprise three subfamilies, which are named an anti-apoptotic family, pro-apoptotic multidomain family, and pro-apoptotic BH3-only protein family, respectively [52]. Bim is one of these BH3-only proteins and its up regulation triggers cytochrome c release from mitochondria, which consequentially induces a chain reaction that entails the formation of the apoptosome and the activation of its effector, caspase-9. Thus, Bim up regulation induces apoptosis [53]. MiR192 is reported to bind to 3'-UTR of Bim mRNA and negatively regulates Bim expression at the post-transcriptional level in lung adenocarcinoma cells, leading to CDDP resistance [54]. Moreover, Let-7c directly interacts with the 3'UTR of Bcl-XL, which is an antiapoptotic protein, and down regulation of Let-7c increases the sensitivity of A549/DDP cells to DDP [55]. Down-regulation of miR-155 can reduce the expression of Bax and then enhance the sensitivity of A549 cells to CDDP treatment via modulating cellular apoptosis and DNA damage through an Apaf-1-mediated pathway [56]. Meanwhile, miR-503, miR-181a and has-miR-497 are also reported to modulate apoptosis via inhibiting Bcl-2 family protein expression [57, 58].

MAPK subfamily members (p38, JNK, and ERK) are intimately associated with the mode of action of CDDP. MKK4 and MKK7, which are required for synergistic JNK activation, are the most common targets for cell death signaling in kinase cascade. Additionally, the activity of JNK is negatively regulated by a group of MAPK phosphatases of which MKP1 is the major JNK suppressor. Interestingly, CDDP induces MKP1 expression, which is assumed to be a cytoprotective response to CDDP in cancer cells. In the above mechanisms, RIP1 (Receptorinteracting protein 1) plays a vital role. A recent study showed that the expression of miR940 was substantially increased in RIP1-knockdown cells, suggesting that RIP1 can release miR-940-mediated suppression of MKP1 expression to suppress JNK activation, attenuating the anticancer activity of CDDP in turn [59]. 


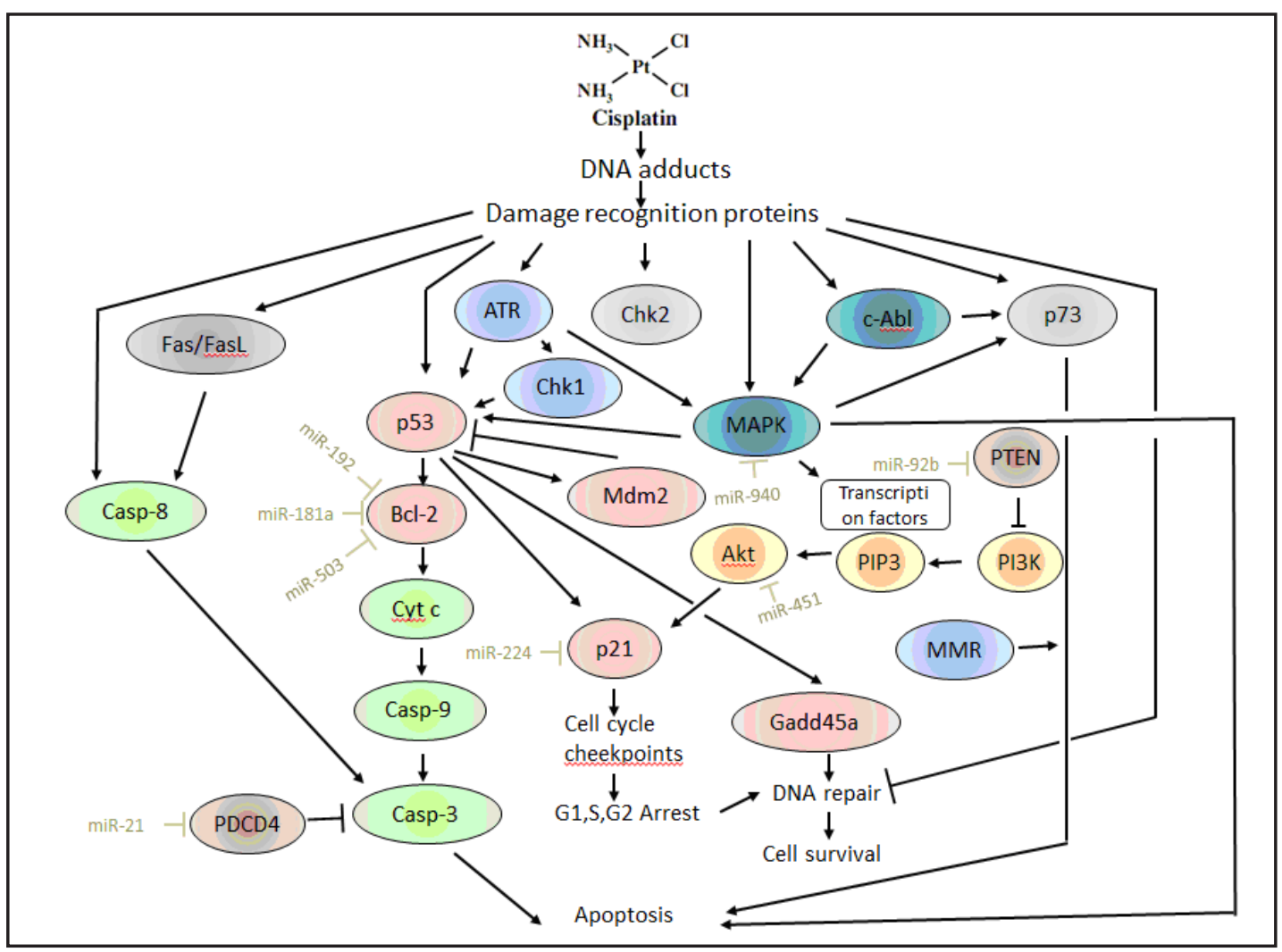

Fig. 3. Involvement of miRNAs in CDDP-induced apoptosis. The apoptotic function of CDDP depends on the signals generated between the intrinsic and the extrinsic apoptosis pathways. The alteration of miRNA expression in tumor cells leads to inhibition of those signaling pathways and then formation of CDDP-resistance through regulation of downstream key target genes and their products.

Phosphatase and tensin homologue (PTEN), which can be targeted by miR-21 or $92 \mathrm{~b}$, inactivates Akt signaling pathway through dephosphorylating phosphoinositide 3-phosphatase (PIP3) and acts as a negative regulator of PI3K/Akt signaling [60, 61]. PI3K/ Akt is also the subsequent downstream mediator of insulin-like growth factor receptors (IGFRs). High miR-139-5P expression is inversely correlated with low expression of insulinlike growth factor 1 receptor (IGF-1R). Inhibition of IGF-1R pathway can regulate the chemoresistance of NSCLC cell lines to CDDP treatment [62, 63]. The antiapoptotic signal may occur as a result of Akt-mediated phosphorylation of procaspase 9, which is then lead to the activation of casapase-3, the ultimate effector casapase. Another suppressor reported to drive apoptosis via activating the casapase-3 pathway is programmed cell death protein 4 (PDCD4). Both miR-21 and miR-182 contribute to the resistance of NSCLC cells to CDDP by target PDCD4 [64]. A recent study demonstrates that overexpression of PDCD4 can increase the sensitivity of cells to CDDP via regulating cell growth. Further research indicates that PDCD4 directly interacts with the DNA binding domain of Twist1, restraining its DNA binding ability and inhibiting Y-box binding protein-1(YB-1) expression [65].

The nucleotide excision repair (NER) pathway is the primary repair pathway involved in repairing CDDP adducts, where ERCC1 is the most important component [66]. Recent studies have shown that the enforced increase of miR-138 levels down-regulates expression of ERCC1 and increases sensitivity of the drug-resistant lung cancer cells to CDDP by inducing apoptosis [67].

Evidences suggest that DNA methyltransferases (DNMTs) can function as an important regulator for epigenetic processes of chemotherapy by regulating apoptosis. In a recent study, Sui' et al. showed a down-regulated of miR-148b expression and evaluated DNMTs expression in CDDP-resisted human NSCLC cell lines compared with their parental NSCLC 


\begin{tabular}{|c|c|c|}
\hline Cellular Physiology & Cell Physiol Biochem 2015;37:1869-1880 & \\
\hline and Biochemistry & $\begin{array}{l}\text { DOI: 10.1159/000438548 } \\
\text { Published online: November 17, } 2015\end{array}$ & $\begin{array}{l}\text { O } 2015 \mathrm{~S} \text {. Karger AG, Basel } \\
\text { www.karger.com/cpb }\end{array}$ \\
\hline
\end{tabular}

Table 1. MiRNAs involved in pathyways affecting cisplatin sensitivity

\begin{tabular}{llllc}
\hline microRNA & Expression & Response to CDDP & Target geen & reference \\
\hline Let-7c & Up & Sensitization & ABCC2 & 43 \\
MIR 17 family & Down & resistance & TGF beta R2 & 36 \\
MIR-21 & Down & Sensitization & PTEN/Bcl-2/PDCD4 & 61 \\
MIR-10a & Down & Sensitization & MDR & 44 \\
MIR-15b & Up & Resistance & PEBP4 & 38 \\
MIR-24-3p & Down & Resistance & ATG4A & 50 \\
MIR-25 & Down & Resistance & Cyclin E2 & 28 \\
MIR-27a & Up & Resistance & RKIP & 39 \\
MIR-31 & up & Resistance & ABCB9 & 42 \\
MIR-34a & Down & Sensitization & PEBP4 / Sirt1 & 22,37 \\
MiR-92b & Up & Sensitization & PTEN & 60 \\
MIR-98 & Down & Sensitization & HMGA2/TP53 & 30 \\
MIR-106a & Up & resistance & ABCA1 & 45 \\
MIR-128-2 & Up & Resistance & E2F5 & 34 \\
MIR-134 & Up & Sensitization & ABCC1 & 46 \\
MIR-137 & Down & Sensitization & KIT & 49 \\
MIR-138 & Up & Sensitization & ERCC1 & 67 \\
MIR-145 & Up & Resistance & CDK6 & 29 \\
MIR-148b & Up & Sensitization & DNMT1 & 68 \\
MIR-155 & Down & Sensitization & Apaf-1 & 56 \\
MIR-182 & up & Sensitization & PDCD4 & 64 \\
MIR-192 & Up & resistance & Bim & 54 \\
MIR-200c & Down & Resistance & ZEB1 /ZEB2 & 35 \\
MIR-217 & Up & sensitization & KRAS & 71 \\
MIR-224 & Up & Resistance & P21 & 33 \\
MIR-451 & Up & Resistance & MDR1 & 42 \\
MIR-495 & Up & Sensitization & ATP7A & 47 \\
MIR-497 & Up & Sensitization & BCL-2 & 58 \\
MIR-503 & Down & Resistance & FANCN /Bcl-2 & 57,72 \\
MIR-940 & Up & Resistance & MKP1 & 59 \\
\hline & & & & \\
\hline
\end{tabular}

cell lines, and further testified that miR-148b reverses CDDP-resistance in NSCLC cells by enhancing apoptosis via negatively regulating DNMT1 expression [68].

\section{Conclusions and Future Directions}

The resistance to CDDP, either de novo or acquired, is a major contributor to the relapse of lung cancer and increases the administered amount of anti-cancer drug agents in turn. While recent researches point to the direct involvement of miRNAs in lung cancer and CDDPresistance, such findings give us a new idea of treatment, which is likely that manipulating relevant miRNAs may be one approach to avoid resistance. Huge evidence shows that specific miRNA can be regulated and then targets downstream genes to re-sensitize cancer cells to the effects of CDDP. For example, the compound (-)-epigallocatechin-3-gallate (EGCG), the major catechin found in green tea, inhibits the expression of hsa-miR-98-5p, followed by an increase of $\mathrm{p} 53$, thus the efficacy of cisplatin is enhanced [69]. Substantial evidence exists 
to indicate that abnormal alterations of expression of miRNAs can be normalized by CTPRFA, highlighting the possibility that combine CDDP-based chemotherapy and CT-PRFA to overcome drug resistance [70]. Due to the trait of miRNAs, it is possible to combine the CDDP-based chemotherapy with miRNA therapeutics to fight lung cancer more effectively. Moreover, the level of some microRNAs in tumor tissues or plasma, such as miR-25, miR-217 and miR-503, correlates with cell resistance to cisplatin [29, 71, 72]. Also, miRNA-21 was reported to be a biomarker predictive for platinum-based adjuvant chemotherapy response in patients with non-small cell lung cancer [73]. This potential ability to predict response to CDDP can help clinical doctors to adjust treatment timely and improve the survival rate of lung cancer. As miRNAs have the prospect to be an adjunct to lung cancer diagnosis and treatment in future, further studies are warranted to elucidate the roles of miRNAs in cellular processes related to CDDP-resistance of tumor cells. It is only a matter of time until miRNA-based therapies is proved to restore the sensitivity of tumor cells to some anticancer drugs including CDDP.

Over recent studies suggest that multiple miRNAs are correlated with development of CDDP resistance in tumor cells. It has been reported that the mechanisms of miRNA regulation include apoptosis, cell cycle, drug transport, or EMT-related signaling pathways, but the exact molecular mechanisms underlying the role of miRNAs in CDDP resistance remain unclear and need to be further elucidated. In this review, we discuss recent studies which demonstrate that miRNAs play a critical role in the regulation of CDDP resistance (Table 1), and targeting specific miRNAs will be an emerging strategy to increase the efficacy of CDDP-based chemotherapy for lung cancer therapy. In the next five years, the molecular mechanisms of miRNAs involved in regulating each of the resistance phenotype will be better understood and miRNA therapeutics will be more promising in clinical applications. With the increasing number of publications in the field, miRNA-based chemotherapy will hold promise for significant improvements in lung cancer treatment on safety permits. It can be seen that in the near future, miRNAs are exciting molecular entities with the potential to overcome CDDP resistance and treat lung cancer more effectively in clinic.

\section{Acknowledgements}

The work was supported by grants from the National Natural Science Foundation of China (No.81172335 and 81472266) and the Excellent Youth Foundation of Jiangsu Province, China (BK20140032).

\section{Disclosure Statement}

The authors declare that they have no conflicts of interest related to this work.

\section{References}

1 Torre LA, Bray F, Siegel RL, Ferlay J, Lortet-Tieulent J, Jemal A: Global cancer statistics, 2012. CA Cancer J Clin 2015;65:87-108.

2 Miller YE: Pathogenesis of lung cancer: 100 year report. Am J Respir Cell Mol Biol 2005;33:216-223.

3 Artal Cortes A, Calera Urquizu L, Hernando Cubero J: Adjuvant chemotherapy in non-small cell lung cancer: state-of-the-art. Transl Lung Cancer Res 2015;4:191-197.

4 Kalemkerian GP: Advances in pharmacotherapy of small cell lung cancer. Expert Opin Pharmacother 2014;15:2385-2396.

5 Siddik ZH: Cisplatin: mode of cytotoxic action and molecular basis of resistance. Oncogene 2003;22:72657279. 


\section{Cellular Physiology Cell Physiol Biochem 2015;37:1869-1880 \begin{tabular}{ll|l} 
and Biochemistry & DOI: 10.1159/000438548 & Published online: November 17, 2015 \\
\hline
\end{tabular} \\ Chen et al.: MiRNAs Involved in Cisplatin Resistance of Lung Cancer}

6 O'Grady S, Finn SP, Cuffe S, Richard DJ, O'Byrne KJ, Barr MP: The role of DNA repair pathways in cisplatin resistant lung cancer. Cancer Treat Rev 2014;40:1161-1170.

7 Zhang B, Zhang K, Liu Z, Hao F, Wang M, Li X, Yin Z, Liang H: Secreted clusterin gene silencing enhances chemosensitivity of a549 cells to cisplatin through AKT and ERK1/2 pathways in vitro. Cell Physiol Biochem 2014;33:1162-1175.

8 Galluzzi L, Senovilla L, Vitale I, Michels J, Martins I, Kepp 0, Castedo M, Kroemer G: Molecular mechanisms of cisplatin resistance. Oncogene 2012;31:1869-1883.

9 MacDonagh L, Gray SG, Finn SP, Cuffe S, O'Byrne KJ, Barr MP: The emerging role of microRNAs in resistance to lung cancer treatments. Cancer Treat Rev 2015;41:160-169.

10 Sarkar FH, Li Y, Wang Z, Kong D, Ali S: Implication of microRNAs in drug resistance for designing novel cancer therapy. Drug Resist Updat 2010;13:57-66.

11 Wan L, Zhu L, Xu J, Lu B, Yang Y, Liu F, Wang Z: MicroRNA-409-3p functions as a tumor suppressor in human lung adenocarcinoma by targeting c-Met. Cell Physiol Biochem 2014;34:1273-1290.

12 Zhang H, Zhang H, Zhao M, Lv Z, Zhang X, Qin X, Wang H, Wang S, Su J, Lv X, Liu H, Du W, Zhou W, Chen X, Fei K: MiR-138 inhibits tumor growth through repression of EZH2 in non-small cell lung cancer. Cell Physiol Biochem 2013;31:56-65.

13 Wu N, Zhang C, Bai C, Han YP, Li Q: MiR-4782-3p inhibited non-small cell lung cancer growth via USP14. Cell Physiol Biochem 2014;33:457-467.

14 Zhang L, Qian J, Qiang Y, Huang H, Wang C, Li D, Xu B: Secreted clusterin gene silencing enhances chemosensitivity of a549 cells to cisplatin through AKT and ERK1/2 pathways in vitro. Cell Physiol Biochem 2014;33:1162-1175.

15 Wang J, Yang B, Han L, Li X, Tao H, Zhang S, Hu Y: Demethylation of miR-9-3 and miR-193a genes suppresses proliferation and promotes apoptosis in non-small cell lung cancer cell lines. Cell Physiol Biochem 2013;32:1707-1719.

16 Xia Y, Chen Q Zhong Z, Xu C, Wu C, Liu B, Chen Y: Down-regulation of miR-30c promotes the invasion of non-small cell lung cancer by targeting MTA1. Cell Physiol Biochem 2013;32:476-485.

17 Lujambio A, Lowe SW: The microcosmos of cancer. Nature 2012;482:347-355.

18 Heo JI, Oh SJ, Kho YJ, Kim JH, Kang HJ, Park SH, Kim HS, Shin JY, Kim MJ, Kim M, Kim SC, Park JB, Kim J, Lee JY: ATM mediates interdependent activation of p53 and ERK through formation of a ternary complex with p-p53 and p-ERK in response to DNA damage. Mol Biol Rep 2012;39:8007-8014.

19 Cheng Q Chen J: Mechanism of p53 stabilization by ATM after DNA damage. Cell Cycle 2010;9:472-478.

20 Chang TC, Wentzel EA, Kent OA, Ramachandran K, Mullendore M, Lee KH, Feldmann G, Yamakuchi M, Ferlito M, Lowenstein CJ, Arking DE, Beer MA, Maitra A, Mendell JT: Transactivation of miR-34a by p53 broadly influences gene expression and promotes apoptosis. Mol Cell 2007;26:745-752.

21 Wang X, Dong K, Gao P, Long M, Lin F, Weng Y, Ouyang Y, Ren J, Zhang H: microRNA-34a sensitizes lung cancer cell lines to DDP treatment independent of p53 status. Cancer Biother Radiopharm 2013;28:45-50.

22 Yamakuchi M, Ferlito M, Lowenstein CJ: miR-34a repression of SIRT1 regulates apoptosis. Proc Natl Acad Sci U S A 2008;105:13421-13426.

23 Skrzypski M, Dziadziuszko R, Jassem J: MicroRNA in lung cancer diagnostics and treatment. Mutat Res 2011;717:25-31.

24 Donzelli S, Fontemaggi G, Fazi F, Di Agostino S, Padula F, Biagioni F, Muti P, Strano S, Blandino G: MicroRNA-128-2 targets the transcriptional repressor E2F5 enhancing mutant p53 gain of function. Cell Death Differ 2012;19:1038-1048.

25 Cao JX, Li SY, An GS, Mao ZB, Jia HT, Ni JH: E2F1-regulated DROSHA promotes miR-630 biosynthesis in cisplatin-exposed cancer cells. Biochem Biophys Res Commun 2014;450:470-475.

26 Cao JX, Lu Y, Qi JJ, An GS, Mao ZB, Jia HT, Li SY, Ni JH: MiR-630 inhibits proliferation by targeting CDC7 kinase, but maintains the apoptotic balance by targeting multiple modulators in human lung cancer A549 cells. Cell Death Dis 2014;5:e1426.

27 Hwang HC, Clurman BE: Cyclin E in normal and neoplastic cell cycles. Oncogene 2005;24:2776-2786.

28 Zhao Z, Liu J, Wang C, Wang Y, Jiang Y, Guo M: MicroRNA-25 regulates small cell lung cancer cell development and cell cycle through cyclin E2. INT J Clin Exp Pathol 2014;7:7726-7734.

29 Bar J, Gorn-Hondermann I, Moretto P, Perkins TJ, Niknejad N, Stewart DJ, Goss GD, Dimitroulakos J: miR Profiling Identifies Cyclin-Dependent Kinase 6 Downregulation as a Potential Mechanism of Acquired Cisplatin Resistance in Non-Small-Cell Lung Carcinoma. Clin Lung Cancer 2015;16:e121-129. 


\section{Cellular Physiology Cell Physiol Biochem 2015;37:1869-1880 \begin{tabular}{l|l|l} 
DOI: 10.1159/000438548 & (C) 2015 S. Karger AG, Basel
\end{tabular} and Biochemistry Published online: November 17, $2015 \quad$ www.karger.com/cpb

30 Zhang S, Zhang C, Li Y, Wang P, Yue Z, Xie S: miR-98 regulates cisplatin-induced A549 cell death by inhibiting TP53 pathway. Biomed Pharmacother 2011;65:436-442.

31 Kuerbitz SJ, Plunkett BS, Walsh WV, Kastan MB: Wild-type p53 is a cell cycle checkpoint determinant following irradiation. Proc Natl Acad Sci USA 1992;89:7491-74955.

32 Smith FM, Stephens RB, Kennedy MJ, Reynolds JV: P53 abnormalities and outcomes in colorectal cancer: a systematic review. Br J Cancer 2005;92:1813.

33 Wang H, Zhu LJ, Yang YC, Wang ZX, Wang R: MiR-224 promotes the chemoresistance of human lung adenocarcinoma cells to cisplatin via regulating G(1)/S transition and apoptosis by targeting p21(WAF1/ CIP1). Br J Cancer 2014;111:339-354.

34 Thiery JP, Sleeman JP: Complex networks orchestrate epithelial-mesenchymal transitions. Nat Rev Mol Cell Biol 2006;7:131-142.

35 Ceppi P, Mudduluru G, Kumarswamy R, Rapa I, Scagliotti GV, Papotti M, Allgayer H: Loss of miR-200c expression induces an aggressive, invasive, and chemoresistant phenotype in non-small cell lung cancer. Mol Cancer Res 2010;8:1207-1216.

36 Jiang Z, Yin J, Fu W, Mo Y, Pan Y, Dai L, Huang H, Li S, Zhao J: MiRNA 17 family regulates cisplatin-resistant and metastasis by targeting TGFbetaR2 in NSCLC. PLoS One 2014;9:e94639.

37 Yu G, Chen G, Huang B, Wu S: Downregulation of PEBP4, a target of miR-34a, sensitizes drug-resistant lung cancer cells. Tumour Biol 2014;35:10341-10349.

38 Zhao Z, Zhang L, Yao Q Tao Z: miR-15b regulates cisplatin resistance and metastasis by targeting PEBP4 in human lung adenocarcinoma cells. Cancer Gene Ther 2015;22:108-114.

39 Li J WY, Song Y, Fu Z, Yu W: miR-27a regulates cisplatin resistance and metastasis by targeting RKIP in human lung adenocarcinoma cells. Mol Cancer 2014;13:193.

40 Zhang F, Li Y, Zhou Q: Advances in the relationship between microRNA and cisplatin resistance of lung cancer. Zhongguo Fei Ai Za Zhi 2014;17:269-272.

41 Demirel O, Bangert I, Tampe R, Abele R: Tuning the cellular trafficking of the lysosomal peptide transporter TAPL by its N-terminal domain. Traffic 2010;11:383-393.

42 Dong Z, Zhong Z, Yang L, Wang S, Gong Z: MicroRNA-31 inhibits cisplatin-induced apoptosis in non-small cell lung cancer cells by regulating the drug transporter ABCB9. Cancer Lett 2014;343:249-257.

43 Zhan M, Qu Q, Wang G, Zhou H: Let-7c sensitizes acquired cisplatin-resistant A549 cells by targeting ABCC2 and Bcl-XL. Pharmazie 2013;68:955-961.

44 Sun W, Ma Y, Chen P, Wang D: MicroRNA-10a silencing reverses cisplatin resistance in the A549/cisplatin human lung cancer cell line via the transforming growth factor-beta/Smad2/STAT3/STAT5 pathway. Mol Med Rep 2015;11:3854-3859.

45 Ma Y, Li X, Cheng S, Wei W, Li Y: MicroRNA-106a confers cisplatin resistance in non-small cell lung cancer A549 cells by targeting adenosine triphosphatase-binding cassette A1. Mol Med Rep 2015;11:625-632.

46 Guo L, Liu Y, Bai Y, Sun Y, Xiao F, Guo Y: Gene expression profiling of drug-resistant small cell lung cancer cells by combining microRNA and cDNA expression analysis. Eur J Cancer 2010;46:1692-1702.

47 Song L, Li Y, Li W, Wu S, Li Z: miR-495 enhances the sensitivity of non-small cell lung cancer cells to platinum by modulation of copper-transporting P-type adenosine triphosphatase A (ATP7A). J Cell Biochem 2014;115:1234-1242.

48 Ali S, Ali S: Role of c-kit/SCF in cause and treatment of gastrointestinal stromal tumors (GIST). Gene 2007;401:38-45.

49 Li P, Ma L, Zhang Y, Ji F, Jin F: MicroRNA-137 down-regulates KIT and inhibits small cell lung cancer cell proliferation. Biomed Pharmacother 2014;68:7-12.

50 Pan B, Chen Y, Song H, Xu Y, Wang R, Chen L: Mir-24-3p downregulation contributes to VP16-DDP resistance in small-cell lung cancer by targeting ATG4A. Oncotarget 2015;6:317-331.

51 Zhou L, Qiu T, Xu J, Wang T, Wang J, Zhou X, Huang Z, Zhu W, Shu Y, Liu P: miR-135a/b modulate cisplatin resistance of human lung cancer cell line by targeting MCL1. Pathol Oncol Res 2013;19:677-683.

52 Youle RJ, Strasser A: The BCL-2 protein family: opposing activities that mediate cell death. Nat Rev Mol Cell Biol 2008;9:47-59.

53 Akiyama T, Dass CR, Choong PF: Bim-targeted cancer therapy: a link between drug action and underlying molecular changes. Mol Cancer Ther 2009;8:3173-3180.

54 Zhang F, Li Y, Wu H, Qi K, You J, Li X, Zu L, Pan Z, Wang Y, Li Y, Li Y, Wang M, Shen W, Zhou Q: MiR-192 confers cisplatin resistance by targeting Bim in lung cancer. Zhongguo Fei Ai Za Zhi 2014;17:384-390. 


\section{Cellular Physiology Cell Physiol Biochem 2015;37:1869-1880 \begin{tabular}{l|l} 
and Biochemistry & $\begin{array}{l}\text { DOI: 10.1159/000438548 } \\
\text { Published online: November 17, } 2015\end{array}$ \\
\hline
\end{tabular} \\ Chen et al.: MiRNAs Involved in Cisplatin Resistance of Lung Cancer}

55 Liu L, Bian K: Advance in studies on molecular mechanisms of cisplatin resistance and intervention with traditional Chinese medicines. Zhongguo Zhong Yao Za Zhi 2014;39:3216-3220.

56 Zang YS, Zhong YF, Fang Z, Li B, An J: MiR-155 inhibits the sensitivity of lung cancer cells to cisplatin via negative regulation of Apaf-1 expression. Cancer Gene Ther 2012;19:773-778.

57 Qiu T, Zhou L, Wang T, Xu J, Wang J, Chen W, Zhou X, Huang Z, Zhu W, Shu Y, Liu P: miR-503 regulates the resistance of non-small cell lung cancer cells to cisplatin by targeting Bcl-2. Int J Mol Med 2013;32:593598.

58 Zhu W, Zhu D, Lu S, Wang T, Wang J, Jiang B, Shu Y, Liu P: miR-497 modulates multidrug resistance of human cancer cell lines by targeting BCL2. Med Oncol 2012;29:384-391.

59 Wang Q, Shi S, He W, Padilla MT, Zhang L, Wang X, Zhang B, Lin Y: Retaining MKP1 expression and attenuating JNK-mediated apoptosis by RIP1 for cisplatin resistance through miR-940 inhibition. Oncotarget 2014;5:1304-1314.

60 Li Y, Li L, Guan Y, Liu X, Meng Q, Guo Q: MiR-92b regulates the cell growth, cisplatin chemosensitivity of A549 non small cell lung cancer cell line and target PTEN. Biochem Biophys Res Commun 2013;440:604610.

61 Xu L, Huang Y, Chen D, He J, Zhu W, Zhang Y, Liu X: Downregulation of miR-21 increases cisplatin sensitivity of non-small-cell lung cancer. Cancer Genet 2014;207:214-220.

62 Tian Z1, Yao G, Song H, Zhou Y, Geng J: IGF2R expression is associated with the chemotherapy response and prognosis of patients with advanced NSCLC. Cell Physiol Biochem 2014;34:1578-1588.

63 Xu W, Hang M, Yuan CY, Wu FL, Chen SB, Xue K: MicroRNA-139-5p inhibits cell proliferation and invasion by targeting insulin-like growth factor 1 receptor in human non-small cell lung cancer.Int J Clin Exp Pathol 2015;8:3864-3870.

64 Ning FL WF, Li ML, Yu ZS, Hao YZ, Chen SS: MicroRNA-182 modulates chemosensitivity of human non-small cell lung cancer to cisplatin by targeting PDCD4. Diagn Pathol 2014;9:143.

65 Shiota M, Izumi H, Tanimoto A, Takahashi M, Miyamoto N, Kashiwagi E, Kidani A, Hirano G, Masubuchi D, Fukunaka Y, Yasuniwa Y, Naito S, Nishizawa S, Sasaguri Y, Kohno K:Programmed cell death protein 4 downregulates Y-box binding protein-1 expression via a direct interaction with Twist1 to suppress cancer cell growth.Cancer Res 2009;69:3148-3156.

66 O'Grady S, Finn SP, Cuffe S, Richard DJ, O'Byrne KJ, Barr MP:The role of DNA repair pathways in cisplatin resistant lung cancer.Cancer Treat Rev 2014;40:1161-1170.

67 Wang Q, Zhong M, Liu W, Li J, Huang J, Zheng L: Alterations of microRNAs in cisplatin-resistant human nonsmall cell lung cancer cells (A549/DDP). Exp Lung Res 2011;37:427-434.

68 Sui C, Meng F, Li Y, Jiang Y: miR-148b reverses cisplatin-resistance in non-small cell cancer cells via negatively regulating DNA (cytosine-5)-methyltransferase 1 (DNMT1) expression. J Transl Med $2015 ; 13: 132$.

69 Zhou DH, WX, Feng Q: EGCG enhances the efficacy of cisplatin by downregulating hsa-miR-98-5p in NSCLC A549 cells. Nutr Cancer 2014;66:636-644.

70 Hu X, Zhang F, Liu XR, Wu YT, Ni YM: Efficacy and potential microRNA mechanism for computed tomography-guided percutaneous radiofrequency ablation of primary lung cancer and lung metastasis from liver cancer. Cell Physiol Biochem 2014;33:1261-1271.

71 Guo J, Feng Z, Huang Z, Wang H, Lu W: MicroRNA-217 functions as a tumour suppressor gene and correlates with cell resistance to cisplatin in lung cancer. Mol Cells 2014;37:664-671.

72 Li N, Zhang F, Li S, Zhou S: Epigenetic silencing of MicroRNA-503 regulates FANCA expression in non-small cell lung cancer cell. Biochem Biophys Res Commun 2014;444:611-616.

73 Gao W, Lu X, Liu L, Xu J, Feng D, Shu Y: MiRNA-21: a biomarker predictive for platinum-based adjuvant chemotherapy response in patients with non-small cell lung cancer. Cancer Biol Ther 2012;13:330-340. 